\title{
Analysis on the Application of Intelligent Detection Technology in Vehicle Performance Testing
}

\author{
Yu Haiyang \\ Institute of Intelligent Manufacturing and Automotive, Chongqing Technology and Business \\ Institute, Chongqing Hechuan,, China, 401520
}

Keywords: intelligent detection technology; vehicle performance detection; application

\begin{abstract}
Nowadays, the motor vehicle market economy is developing rapidly, improving people's quality of life and material taste. Cars bring convenience to people's travel, so people's demand for cars have risen unprecedentedly. However, the continuous increase in the number of cars has increased the traffic congestion and the malfunction of the operation of the automobile device has been greatly increased. In the application process, it is necessary to pay special attention to automotive maintenance work, after further innovation of automotive maintenance technology, and improve the level of automotive maintenance, which is very important for automotive maintenance work.
\end{abstract}

\section{Introduction}

The problem of automotive failure has reduced the stability and comfort of the car to some extent. With the development of the automobile industry, various new technologies have been developed, which has stimulated the forward momentum of the automotive industry. In the automotive system, intelligent and automated technology is widely used, bringing convenience to automotive inspection work. The use of intelligent technology in vehicle detection can make drivers more aware of the performance of the car and effectively respond to emergencies.

\section{Automotive Automation Test Diagnostic Significance}

Integrating intelligent technology into the automotive diagnostic process can improve diagnostic efficiency, find the source of the fault, and achieve automation and intelligent diagnostic goals. In general, vehicle fault diagnosis can start from the following two points:

First, prepare the vehicle for maintenance in advance. Detecting vehicle faults and arranging repairs quickly is the main process of automobile maintenance at present, In order to stabilize the performance of the vehicle, the problem should be detected as early as possible, especially before the failure occurs, do a series of prevention work to provide accurate data information for post-processing. Automotive parts use time and wear degree is very different, so it can play a key role in maintenance, but by other factors, such as material performance, installation adjustment, processing quality, load size, etc., will reduce vehicle performance. The use of intelligent detection technology can reduce vehicle maintenance time, increase the speed, and provide guarantees for various performance indicators of automotive. 
Second, the maintenance efficiency is accelerated. Using intelligent detection technology can reduce the number of car disassembly. When the vehicle is inspected, use modeling to explore and deal with problems, combine it with life, choose the appropriate maintenance plan, and speed up the maintenance efficiency. In addition, in order to promote the development of the automotive industry, it is necessary to build a comprehensive performance testing station for automobiles, monitor the quality of vehicle maintenance in real time, and conduct random inspections to improve the level of vehicle maintenance.

\section{Automotive Inspection Technology Classification}

Nowadays, China's automotive ownership has increased significantly. Therefore, the safety performance inspection and testing of automotive is an important development goal and direction of automotive detection technology, and it is also an important core component to ensure safe driving of automotive.

\subsection{Automotive Performance Testing}

Automotive performance testing refers to comprehensive testing of various vehicle performances, overall detection of vehicle operating conditions and technical functions, exploration of vehicle failure problems, real-time monitoring of vehicle quality, enhancement of overall vehicle stability and economic performance, and improvement of corporate economic theory. Vehicle safety and environmental protection testing refers to the main observation of whether the car is driving normally and whether the environmental protection work is qualified. The car does not need to be dismantled during safety and environmental protection testing. It is completed by means of safety and environmental protection monitoring system, so that the overall performance of the car meets the standard specifications, reducing exhaust emissions and noise pollution, and creating a high-efficiency, low-pollution driving environment for people. The main purpose of automotive inspection technology is to quickly and easily infer and predict vehicle performance and driving conditions. At present, China's automotive inspection technology is constantly developing, making inspection equipment widely used in various regions, and gradually moving toward autonomous and intelligent direction. Compared with the vehicle detection technology, the direction is to improve the detection system and monitoring system, replace the artificial machinery detection method, further explore the efficacy of the vehicle monitoring system, and use the vehicle prediction skills in the internal parts of the vehicle to make the inspection data more precise, reasonable, extend the life of the car and improve safety.

\subsection{Testing Technology Standardlization}

According to the process of automotive inspection technology, people pay too much attention to some hardware technologies. The research on low-efficiency and difficult testing techniques are not thoroughly studied by the state and government. For the testing equipment, the technical standardization is poor, which makes the automotive have obvious errors in the detection. Therefore, it is necessary to innovate hardware technology software on a regular basis so that it conforms to the relevant national standards system. The detection methods and technologies of each region are consistent, and the requirements and specifications are unified to improve the accuracy and safety of vehicle detection. 


\subsection{Testing Equipment Standardization}

Automotive inspection technology has a long history of development, from small to large, from nonexistence to pass into existence. The initial stage is to introduce new vehicle detection technology and equipment from developed countries, and put them into operation after research and analysis. Although China's automotive inspection technology has developed very well and achieved good results, it still has a long way to go compared with advanced countries. In terms of the current form, despite the large number of automotive testing equipment, some equipments have poor accuracy, quality and practicality, which are inconsistent with automotive testing standards. In this regard, the state needs to pay attention to the construction of automotive inspection technology software, which is the core content of the later development, in line with national standards, further research on testing equipment, and make the detection technology move toward the standardization direction.

\subsection{Rationalization of Detection Methods}

Vehicle inspection stations need to be strictly managed during the inspection process to ensure the authenticity of the test results, which is recognized by the public. The vehicle inspection station must be approved by the government, and the work content is to supervise the inspection technology to meet the standards. At the same time, the automotive company must also invest a lot of technology, the inspection station investors include government transportation, private construction. Its equipment and staff need to pass a professional review. The vehicle inspection and operation station should regularly hold an annual inspection system, which is mainly divided into two categories: spot check and appraisal. Spot check for infringing vehicles, vehicles with serious violations must be revoked and financially punished; appraisal is to verify vehicle detection technology.

\subsection{Intelligent Automotive Detection}

At present, computer monitoring and detection method is widely used in domestic automobile detection system, although these two detection methods are very novel, the detection results are also more accurate, but because the management is not consistent, this makes the detection station have significant differences in the use of monitoring and detection methods. Even with the use of the most innovative network information technology at present, only one detection station can be made intelligent, and intelligence cannot be completed among other inspection stations. With the rapid development of information technology, we will definitely explore ways to solve this problem. In the automotive industry, the testing system staff has strong professional skills, rich work experience, and familiar with basic legal knowledge. However, according to the current situation, it is not satisfactory. The experienced staff, their knowledge and skills are lagging behind, the new employees have strong professional knowledge and skills, but lack of experience makes it difficult for the work to proceed smoothly. Therefore, the overall technical level of the enterprise is poor. If the system fails, there are very few employees that can be dealt with, which will seriously affect the user and the vehicle detection line. In order to reduce the problems caused by hardware and equipment problems for users and enterprises, enterprises should pay great attention to the work of fault diagnosis of test lines, to some extent, no matter any enterprise system will have failure problems, the automatic performance detection system is also the same. Despite the use of new techniques in the design phase, the system will have problems, and the difference is the number of times the problem occurs. Therefore, further research on the theory and use of fault diagnosis has been highly valued. The main factor is that it has a certain impact on the safety, applicability and 
stability of system testing. The rapid development of information technology has improved the level of automation to a certain extent. The function of the automatic detection system of the automotive is also more complete. At the same time, the system has become very complicated, which has increased the difficulty of operation and maintenance in the later stage, resulting in high system maintenance costs. Not only that, If affected by external factors, the system will be shut down to check, the consequences are self-evident. Therefore, it is necessary to upgrade the system on time, strengthen the system security and practicability, improve the system reparability, and provide high-quality services for automotive fault technology.

\section{Development Situation of Automotive Inspection Technology}

Nowadays, the key direction of the future development of China's automotive industry testing equipment is reflected in the following two points: First, the rapid development of information technology has promoted the development of automotive technology to a certain extent, testing instruments are gradually moving towards integration and electronic direction. At this stage, electronic parts are the most widely used components in automotive systems, with a certain diversified characteristics, and the number is also rising sharply, whether it is the machinery used in vehicle maintenance, or the detection of automotive exhaust equipment, or the diagnosis and analysis of vehicle failure equipment, are different from traditional mechanical operations, but gradually moves toward intelligent diagnostic equipment and analysis. In particular, the internal detection and fault diagnosis of the vehicle needs to be completed by using other electronic testing instruments. The development of electronic technology is inseparable from the driving of information technology. Compared with electronic technology, it has diversified development situation in various types of automotive testing instruments, which has improved the accuracy and practicability of automotive maintenance testing equipment. Secondly, the arrival of modern economic society, the testing instruments are gradually moving towards the direction of intelligence and digitization. People's demand for vehicle performance is increasing, such as fault diagnosis systems, navigation systems, etc., and the speed is gradually increasing with the development of the industry, making the vehicles performance and quality technologies have achieved significant results, while at the same time, further research on vehicle testing equipment to improve its performance, which can greatly improve vehicle quality and production technology, develop and use engine diagnostic technology and four-wheel directional inspection equipment, Promote the development of vehicle detection equipment in the direction of intelligence and automation.

\section{Application of Intelligent Detection Technology in Vehicle Performance Testing}

In the automotive system, the use of intelligent technology is very wide, such as in the automotive control system, automotive inspection system, can complete the intelligent and automated management tasks, further improve the level of vehicle performance control, and enhance the stability and reliability of the vehicle.

\subsection{Sensor System Troubleshooting}

In the intelligent vehicle performance testing, the sensor system is its core element, in the automobile application stage to detect all kinds of parts, the use of sensor system can quickly know the operation of other parts of the vehicle, to facilitate the driver to clearly grasp the driving status of the vehicle, better response to emergencies, solve the problem, prevent the occurrence of other accidents. The automotive sensor system occupies the main position in the whole system and plays the role of supporting detection. It is responsible for collecting and transmitting various types of 
signals. The main collected signals are data such as vehicle driving frequency, oil temperature, dry humidity and driving distance. In the process of statistical data, a comprehensive analysis of the data is required. If the problem data is found, it must be resolved as soon as possible. Because of the sensor's own characteristics, once the sensor has a problem, it is not necessary to find another route, as long as the software to write sensor input signal recognition program, the sensor fault automatic diagnosis can be completed. When the sensor is in working condition, it can sense the signal, then send out the signal, and then the signal will appear in the control system. The control system should use the detection software to diagnose whether there is a line fault in the sensor according to the standard value set in advance.

\subsection{Microcomputer System Fault Diagnosis}

The meaning of the microcomputer system in the whole vehicle system is very significant, and it occupies a certain proportion. If the vehicle microcomputer system fails, it will affect the normal operation of the system control program, resulting in the car not being able to drive normally. The automotive microcomputer system often has faults, so it is necessary to solve the problem of car microcomputer system failure as soon as possible, the standby integrated circuit system is placed inside the microcomputer system, as long as the automobile microcomputer system fails, the system will automatically connect the standby integrated circuit, complete the vehicle performance and spare parts control work. In addition, if the automotive microcomputer system caused the failure, the microcomputer automatic diagnosis system can identify the microcomputer system failure, and then display to the interface, record the fault code, when the fault light flashes, is to remind the driver of the car microcomputer system failure, need to solve as soon as possible. Inside the vehicle control system, a device for monitoring the operation of the microcomputer system is installed. This device is called a monitoring loop circuit, and a monitor is provided in the monitoring loop circuit, and the microcomputer system works independently without mutual interference. If the vehicle microcomputer control system is in a normal state at all times, the program running inside the system will accurately record the information in the monitor to prevent the contents of the monitor from leaking. However, if the microcomputer control system fails, the level of the transmission will change, from low to high, triggering the backup circuit, the backup circuit controls the injector and igniter with the aid of a corresponding program.

\subsection{Actuator Troubleshooting}

The actuator plays an important role in the vehicle control system and has a very important meaning. The actuator directly affects the driving state and operation of the vehicle. However, during the running phase of the actuator, other parts will produce some faults, so it is necessary to use intelligence to solve the fault in the actuator. If the car actuator fails, the fail-safe system will fully demonstrate its role. The fault diagnosis module will formulate a series of methods to stop the other functions of the car and keep the car out of operation. This process is called fail-safe. For the ignition device of a vehicle, when the fault occurs, the gas without combustion and incomplete combustion will be discharged out of the pipe, adding the appropriate catalyst will cause the ignition device to malfunction. In the intelligent control system, the fault insurance system will receive the fault signal from the igniter, and then turn off the fuel injection system power supply, terminate the fuel injection work, and provide guarantee for the safety of the vehicle.

\section{Conclusion}

To sum up, nowadays, the motor vehicle market economy is developing rapidly, improving 
people's quality of life and material taste. Cars bring convenience to people's travel, so people's demand for cars have risen unprecedentedly. At present, intelligent technology and automation technology are widely used in various fields of the world. Innovative technology plays a dominant role in the social development stage and is a key factor driving the rapid development of various industries. In the performance testing of automotive, it is necessary to use intelligent detection technology to fundamentally improve the level of vehicle performance detection, so that the driver can better understand the performance of the vehicle, effectively respond to emergencies, and at the same time provide convenience for the later vehicle maintenance work.

\title{
Acknowledgement
}

\author{
Science and Technology Extension Project of Chongqing Education Commission \\ (GZTG201614).
}

\section{References}

[1] Zhu Hui, Xu Jingyang. Application Analysis of Computer Detection and Control Technology in Automotive Electronic Control Devices [J]. Shandong Industrial Technology, 2016(1):159-160.

[2] Hou Bin. Analysis on the Development of Automobile Inspection Technology [J]. China High-tech Zone, 2017(06):63-64.

[3] Wang Chen. Application of Intelligent Automation Technology in Automobile Engineering [J]. Internal combustion engine and accessories, 2017(15):301-302.

[4] Ran Jianguang. Application Analysis of Modern Electronic Technology in Automobile Intelligent Management System [J]. Electronic test, 2016(2):56-57.

[5] Li Xiangnan. Analysis on the Development of Automobile Detection and Diagnosis Technology in China [J]. Scientific consultation, 2016(44):87-88.

[6] Sun Jianhua. Comprehensive Performance Testing and Maintenance Technology for Automotive Applications [J]. Car and driving maintenance (repair version), 2017(11):70-71.

[7] Ye Tao. Application of Electronic Detection Technology in Automobile Detection [J]. China High-tech Zone, 2017(04):72-73.

[8] Ding Hong. The role of electronic technology in the improvement and detection of automobile safety performance [J]. Electronic world, 2016(13):85-86.

[9] Jia Hongjuan. Research on the Status Quo and Development Trend of Automobile Inspection and Diagnosis Technology [J]. Science and wealth, 2015(25):80-81.

[10] Lin Kechun. Research and Simulation Analysis of Automobile Braking Performance Testing System [J]. Journal of Liaoning Institute of Science and Technology, 2017(2):188-189. 